\title{
Linear Colliders Driven by a Superconducting Linac-FEL System
}

\author{
U.Amaldi ${ }^{1)}$ and C.Pellegrini ${ }^{2}$
}

Geneva, 26 August, 1986

Abstract: In this paper we discuss linear colliders in the TeV energy region, based on a two beam accelerator scheme. The low energy beam is used in a Free Electron Laser to produce short wavelength radiation, in the range from one $\mathrm{cm}$ to a fraction of a $\mathrm{mm}$. The energy lost by this beam is restored by a superconducting linac. The short wavelength radiation is fed to a high frequency, linac-type structure, where the high energy beam is accelerated. We give a review of the scaling laws for a linear collider and use them to find some possible set of parameters for our system. We then discuss some of the accelerator technical problems and the beam physics problems encountered in the design of such a system.

\footnotetext{
1) CERN, Geneve, Suisse

2) on leave from Brookhaven National Laboratory, Upton, N.Y.
} 


\section{Introduction}

Particle accelerators have been built and used for about 50 years. During this time their energy has increased from the $\mathrm{MeV}$ to the $\mathrm{TeV}$ region, using at each energy step new and innovative ideas and techniques. Of the next electron positron colliders, like Tristan, LEP and SLC, now being built, LEP (100 GeV, $4.3 \mathrm{~km}$ radius) is probably the last member of a family of accelerators, the electron-positron storage rings, which starting from AdA (250 MeV, $1 \mathrm{~m}$ radius) in the early 60's, has evolved through ACO, Adone, Vep2 and 3, Spear, DCI, CESR, PEP and Petra and has given many important contributions to high energy physics.

It was pointed out by Richter (1) that, because of synchrotron radiation losses, the radius of an electron storage ring scales like the square of its energy. This makes difficult and uneconomical the construction of rings at energy larger than that of LEP.

The next family of electron-positron colliders is that of "linear colliders" (2), of which SLC (50 GeV, length $3 \mathrm{~km})$ is the first member. We hope that an extension of the ideas, physical principles and techniques that are under test in the SLC will allow us to build colliders in the TeV region, with luminosity much larger than $10^{32} / \mathrm{cm}^{2} / \mathrm{s}$, as required by the expectation of the point-like cross-section which drops as $1 / E^{2}$.

In this paper, we discuss some of the problems facing us in the design and construction of a high luminosity linear collider in the $\mathrm{TeV}$ region. The scaling laws for this system will be discussed in section 2 . This will help 
us in understanding what are the characteristics needed for the electron and positron beams. As has already been discussed by many authors $(2,3,4,5,6)$, the two most important effects limiting the collider performance are the "disruption" (7) and the "beamstrahlung" $(8,9,10)$. In particular the beamstrahlung determines two different regimes of operation ${ }^{(8)}$, the "quantum" and the "classical" regime, defined by the condition that the radiation critical energy is larger or smaller than the beam energy. To reduce the beam power, and the total power needed to operate the system, is necessary to work in the quantum regime. This becomes more so when going to energies of several TeV or luminosities in the $10^{34} \mathrm{~cm}^{-2} \mathrm{~s}^{-1}$ range.

In sections 3 and 4 we relate these beam characteristics to the accelerator characteristics, like operating frequency and acceleration rate. In these sections we show that there is a relationship between the choice of the regime of operation, quantum or classical, and the choice of the accelerator frequency. In particular the quantum regime requires the use of accelerators operating at wavelength in the millimiter or submillimeter range, while in the classical regime one has to use wavelength around one centimeter. We believe this to be very important because the development of colliders in the range of $5 \mathrm{TeV}$ and $10^{34} \mathrm{~cm}^{-2} \mathrm{~s}^{-1}$ will force us in the quantum regime and so in the millimetric waves.

To illustrate these results we will discuss, in sections 5,6 and 8 , a two-beam accelerator scheme based on a low frequency superconducting linac, which feeds energy to an electron beam used to operate a Free Electron Laser. The radiation produced in the Free Electron Laser is then used to accelerate a high energy beam to the $\mathrm{TeV}$ energy range, in a high fre- 
quency Linac-type structure, following an idea proposed by Sessler ${ }^{(11)}$. We will consider two examples, at $(1+1)$ and $(5+5) \mathrm{TeV}$. For each one we consider a collider in the classical regime using a linac operating at about $30 \mathrm{GHz}$, and one in the quantum regime operating at about $700 \mathrm{GHz}$. We will show that our sheme allows to reach accelerating fields in the range .1 to $1 \mathrm{GeV} / \mathrm{m}$.

The damping rings needed to produce the electron and positron beams with the emittance required for high luminosity are discussed shortly in section 7 . In the last section we discuss our conclusion and point out the many problems that need to be studied to obtain a full understanding of this collider.

\section{Scaling Laws}

The main formulae relating the beam current and current density to the collider luminosity and other important parameters, have been discussed by several authors (see, for instance references 3 to 6 ). For the convenience of the reader we will summarize them again, limiting ourselves for simplicity to the case of electron and positron beams with cylindrical symmetry. The discussion of the case of a flat beam and of its possible advantages can be found in ref. (4).

For cylindrical bunches of $\mathrm{N}$ electrons or positrons, having transverse and longitudinal gaussian distributions with r.m.s. radius $\sigma_{t}$, and length $\sigma_{1}$ respectively, the luminosity is 


$$
\mathscr{L}=\frac{f N^{2} H}{4 \pi \sigma_{t}^{2}}
$$

where $f$ is the number of bunch crossing per second and $H$ is an "enhancement factor", describing the self focusing of the electron and positron bunches during the collision.

When crossing, each bunch produces a focusing force on the particles of the other bunch. The resulting deviation is described by the disruption parameter, $D$, given by

$$
D=\frac{r_{e}^{N \sigma_{1}}}{\gamma \sigma_{t}^{2}} \equiv \frac{\sigma_{1}}{F}
$$

where, in the small $D$ case, $F$ is the focal distance of a focusing lens equivalent to the bunch, $r_{e}$ is the classical electron radius and $\gamma$ is the electron energy, $E$, in rest mass units, $E=y m c^{2}$. This focusing of the bunches produces a pinch effect, which increases the bunch density and hence the luminosity. On the other hand if the focusing becomes too large, it can lead to a beam filamentation and a decrease in luminosity. This effect is described by the enhancement factor, $H$, which is a function of the disruption parameter D. This focusing and pinch effect has been studied by Hollebeek (7). His results show that up to values of the disruption parameter, D, of the order or smaller than one, the luminosity can be calculated 
ignoring this effect $(H \approx 1)$; for $D$ increasing from one to two the luminosity is enhanced by the pinch effect and one can gain up to a factor of 6 $(H \approx 6)$; for $D$ larger than two, the luminosity tends to remain constant and then to decrease.

A quantity very important in determining the costruction and operation cost of a collider is the average beam power, $P$, related to the particle energy, $E$, by

$$
P=f N E
$$

The next important effect is beamstrahlung, describing the energy loss of an electron caused by the emission of synchrotron radiation in crossing the high magnetic field produced by the other bunch. Following the work of references $4,8,9,10$ we can characterize this effect with two of three related parameters, namely the ratio, $\Upsilon$, of the critical photon energy, $E_{c^{\prime}}$ to the beam energy $\left(\Upsilon=2 E_{c} / 3 E\right)$; the fluctuation parameter $\Gamma$; and the beamstrahlung parameter $\delta$, which equals the average fractional energy loss in the limit $r<<1$ (12). In the limit $r<<1$ the beamstrahlung spectral intensity can be described using the "classical" synchrotron radiation spectrum, which is nearly flat up to the critical photon energy and then decreases exponentially. When $r>>1$ quantum corrections to the synchrotron radiation spectrum become important and the photon spectrum is cut off at the beam energy. This is the so called quantum regime.

The synchrotron radiation critical energy $E_{c}$ is such that 


$$
r=\frac{r^{h c y N \vee H}}{\sqrt{ } 2 m c^{2} \sigma_{t} \sigma_{1}}
$$

The fluctuation parameter is

$$
\Gamma=\frac{r_{e} \gamma}{\alpha_{e m} \sigma_{1}}
$$

where $\alpha_{\text {em }}=1 / 137$. As noted by $J$. Sandweiss, the quantity $\Gamma$ is equal, in the electron rest frame, to the ratio $D E / \mathrm{mc}^{2}, D E$ being the uncertainty in the electron energy produced by the finite interaction time $\Delta T=\sigma_{1} / 2 \gamma c$. The last parameter is

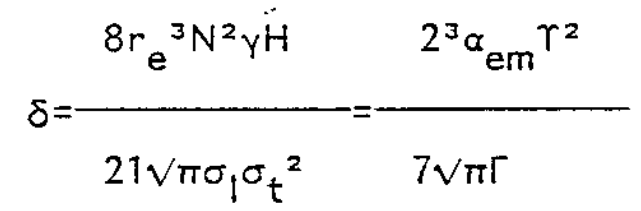

The last equality shows that only two of the three parameters $\Upsilon, \Gamma$, and $\delta$, are independent.

The average fractional energy loss, $\langle\varepsilon\rangle=\langle\Delta E / E\rangle$, is given by

$$
\begin{aligned}
& \langle\varepsilon\rangle=\delta, \text { for } \gamma\langle\langle 1 \\
& \langle\varepsilon\rangle=(2 / 3)^{1 / 3} \delta / \Upsilon^{4} / 3 \text {, for } \Upsilon>>1
\end{aligned}
$$


Note that a factor $1 / 2$ was missing in the equation of ref. (9) corresponding to eq. (8), as pointed out by Yokoya (10).

There are two other quantities that are of interest in evaluating the beamstrahlung effect; one is the average number of photons emitted per crossing (10)

$$
\begin{array}{ll}
N_{Y}=8\langle\varepsilon\rangle / 3 \gamma & , \text { for } \gamma\langle<1 \\
N_{Y}=4\langle\varepsilon\rangle & , \text { for } \gamma>>1
\end{array}
$$

and the other is the r.m.s. electron energy spread

$$
\begin{array}{ll}
\sigma_{\varepsilon}=(\langle\varepsilon\rangle / \sqrt{ } 2 \pi)\left\{1+31.3 / N_{Y}\right\}^{1 / 2}, & \text { for } r<<1 \\
\sigma_{\varepsilon}=.19\langle\varepsilon\rangle\left\{1+64.8 / N_{Y}\right\}^{1 / 2}, & \text { for } r>>1
\end{array}
$$

Of the two terms appearing in $\sigma_{\varepsilon^{\prime}}$ the first represents the energy spread due to to the change of the energy loss with the particle trajectory ( a particle crossing the other bunch on axis has zero energy loss, while one crossing on the edge see the largest magnetic field and has the largest loss), the second describes the effect of the fluctuation in the number of emitted photons. Note that the energy distribution due to beamstrahlung has typically a peak very close to the beam energy $E$, and a long tail at smaller energy. In these circumstances the r.m.s. spread $\sigma_{\varepsilon}$ has a doubtful meaning. 
Although we do not have any analytical result for the average energy loss in the intermediate cases, $\Upsilon \approx 1$, we will use in our calculations simple interpolating formulae, as done in ref. (4),

$$
\langle\varepsilon\rangle=\delta F(\Upsilon)
$$

with the function $F(\Upsilon)$ given by

(4)

1

$$
F(\Upsilon)=\frac{\left(1+(3 / 2)^{1 / 4} \Upsilon\right)^{4 / 3}}{(1+3)}
$$

Similarly we take

$$
N_{Y}=4\langle\varepsilon\rangle(2+3 \Upsilon) / 3 \Upsilon
$$

In designing a collider we want to obtain a certain luminosity for a given beam power, disruption and average beamstrahlung energy loss. The system is described by ten parameters $\left(E, L, P, D,\langle\varepsilon\rangle, \Upsilon, f, N, \sigma_{t}, \sigma_{1}\right)$ and the five equations (1),(2),(3),(4), (13), relate them. Hence one can specify five parameters and compute the others. If we use the five parameters $E$, $\mathscr{L}, P, D,\langle\varepsilon\rangle$, (and remember that $H$ is determined by $D$ ), we obtain the relationships 


$$
\begin{aligned}
& \sigma_{1}=\left(1 / 4 \pi r \mathrm{e}^{m c^{2}}\right)(\mathrm{HDP} / \mathscr{L}) \\
& \mathrm{f}=\left(1 / \mathrm{C}_{1}\right)\left(\mathrm{EL} \mathscr{L}^{2} F(\Upsilon) / \mathrm{PHD} \varepsilon \varepsilon\right) \\
& \mathrm{N}=\mathrm{C}_{1}(P / E \mathscr{L})^{2}(\langle\varepsilon\rangle / F(\Upsilon)) H D \\
& \sigma_{t}^{2}=\left(C_{1} / 4 \pi\right)(P / E \mathscr{L})^{3}(\langle\varepsilon\rangle / F(\Upsilon)) D H^{2}
\end{aligned}
$$

where

$$
C_{1}=21 /\left(2^{7} \pi^{3 / 2} r_{e^{4}}\right)
$$

and the ratio of critical to beam energy is obtained by solving the equation

$$
\Upsilon^{2} F(\Upsilon)=\left(7 \pi^{3 / 2} r_{e}^{\left.2 / 2 \alpha_{e m}{ }^{2}\right)(E \mathscr{L}\langle\varepsilon\rangle / P H D)}\right.
$$

obtained from (1), (5), (6), (13), (16). This equation can be solved by introducing the "quantum parameter"(4)

$$
q=\left(r_{e} / 2 \alpha_{e m}\right)\left(294 \pi^{3}\right)^{1 / 4}(E \mathscr{L}\langle\varepsilon\rangle / P H D)^{1 / 2}
$$

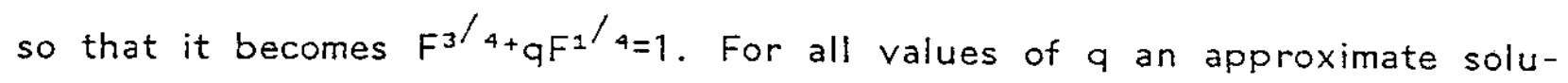
tion, to better than $5^{\circ}$, is $(4)$

$$
F(\Upsilon)=F(q)=\left\{1+4 q(1+4 q / 5) / 3+q^{4}\right\}^{-1}
$$


which allows to express directly Eqs. 16-19 as functions of $E, \mathscr{L}, P, D$, $\langle\varepsilon\rangle$.

Eq. (23) displays very clearly the two regimes. For $q<<1$, classical regime, the $q^{2}$ and $q^{4}$ terms in (23) are engligible, and $F \approx 1-4 q$. For $q>>1$, quantum regime, the $q^{4}$ term is dominant, and $F \approx 1 / q^{4}$. Using (18) and (23) one can then obtain a simple expression for the number of electrons, $N_{Q^{\prime}}$ in the quantum limit

$$
N_{Q} \approx(7 \sqrt{ } \pi)^{3}\left(3 / 32 \alpha_{e m}\right)^{2}\left(\langle\varepsilon\rangle^{3} / H D\right)
$$

We will use (24) later on in discussing the accelerator system.

Instead of $\sigma_{t}$ it is often useful to introduce the normalized beam emittance $\varepsilon_{n}$

$$
\varepsilon_{n}=\gamma \sigma_{t}^{2 / \beta^{*}}
$$

where $\beta^{*}$ is determined by the optical system focusing the beam at the collision point. Not to unnecessarily reduce the luminosity, we must require that $\beta^{*} \geq 3 \sigma_{1}$. Once $\beta^{*}$ is given, eqs. (19), (23) and (25) can be numerically solved for $D$ as a function of $\varepsilon_{n}$ and of the other main parameters $E$, $\mathscr{L}, P,\langle\varepsilon\rangle$. This is relevant in the quantum regime, where $D$ is usually small and well below its upper limit $D \approx 2$, while the emittance becomes very small and $\varepsilon_{n}$ plays the role of a limiting parameter. 
To illustrate the scaling laws we have considered two colliders, one with $\mathrm{E}=1 \mathrm{TeV}, \mathscr{L}=10^{33} \mathrm{~cm}^{-2} \mathrm{~s}^{-1}$, the second with $\mathrm{E}=5 \mathrm{TeV}, \mathscr{L}=10^{34} \mathrm{~cm}^{-2} \mathrm{~s}^{-1}$. In figures 1 to 4 we give the collision frequency, number of electrons/bunch, transverse and longitudinal r.m.s. bunch size versus beam power. In all cases we have assumed the same average energy loss, $\langle\varepsilon\rangle=.08$, and have changed the disruption parameter from a high, $D=1.3$, to a low, $D=.05$, value.

The choice of one particular set of parameters must take into account the technical limitations existing for some quantities, like for instance the minimum transverse beam radius. This cannot be made smaller than a value determined by the beam emittance and by the final focusing system. Some of these limitations are not yet fully understood, so that any choice of parameters done now is necessarily only tentative.

In this spirit we consider now in more details two colliders, one for $(7+1) \mathrm{TeV}, \mathscr{L}=10^{33} / \mathrm{cm}^{2} / \mathrm{s}$, another for $(5+5) \mathrm{TeV}, \mathscr{L}=10^{34} / \mathrm{cm}^{2} / \mathrm{s}$. Even larger values of the luminosity might be needed to compensate the decrease in $e^{+}-e^{-}$reaction cross section with increasing center of mass energy (4), but we shall see later that this implies very difficult conditions for some of the accelerator parameters. A low value of the average beamstrahlung energy loss is chosen in order to limit the particle energy spread in the initial state and not to throw away too much of the energy given to the beam.

The sets of parameters given in Table 1 describe examples of possible colliders, and are not intended to represent optimized systems. They can however be useful to illustrate some of the problems that will be encoun- 
tered in the construction of such systems. For these reasons we have considered colliders working in the classical regime, and in the quantum regime. These numbers will also be used to evaluate the characteristics of our FEL-driven linac.

We have chosen these examples using the following criteria:
a) among the five input parameters discussed earlier we keep constant E, $\mathscr{L},\langle\varepsilon\rangle$;

b) when we reduce the power for constant luminosity, we have to compensate the reduced number of particles by squeezing down the beam radius, i.e. decreasing the emittance; since at the present stage of development we do not know how small a beam emittance can be produced we have chosen our three cases with an emittance smaller by about a factor of four than that obtained in SLC damping rings, for case 1, and smaller by about two order of magnitudes in cases 2 and 3; a smaller emittance would lead to an even smaller beam power;

c) when reducing the emittance we are also forced by (2) and (4) to go in a regime with smaller disruption and larger ratio of critical to beam energy; this fact is also reflected in Table 1, where one can see that case 1 is "classical" $(\Upsilon<1)$ and cases 2 and 3 are "quantistical";

d) we have not given a "classical $(5+5) \mathrm{TeV}$ collider because it would require either an emittance smaller by another order of magnitude, and/or much larger beam power, so that we consider it impractical (the problems are apparent from Figs. 3 and 4 ).

The numbers appearing in Table 1 require some further comments. The first point is on the beam power; the real power needed to operate a sys- 


\section{Table 1: Colliders Parameters}

\begin{tabular}{|c|c|c|c|}
\hline & "Classical" & "Quantum" & "Quantum" \\
\hline luminosity $\left(\mathrm{cm}^{-2} \mathrm{~s}^{-1}\right), \mathscr{L}$ & $10^{33}$ & $10^{33}$ & $10^{34}$ \\
\hline energy $(\mathrm{TeV}), \mathrm{E}$ & 1 & 1 & 5 \\
\hline fractional energy loss, $\langle\varepsilon\rangle$ & .08 & .08 & .08 \\
\hline beam power $(M W), P$ & 10 & 3 & 6 \\
\hline disruption parameter, D & 1.3 & .05 & .015 \\
\hline enhancement factor, $\mathrm{H}$ & 4.5 & 1 & 1 \\
\hline electrons/bunch, $\mathrm{N}$ & $9.8 \times 10^{9}$ & $1.1 \times 10^{8}$ & $2.0 \times 10^{8}$ \\
\hline frequency, $\mathrm{Hz}, \mathrm{f}$ & $6.4 \times 10^{3}$ & $1.7 \times 10^{5}$ & $3.7 \times 10^{4}$ \\
\hline r.m.s. bunch length $(\mathrm{mm}), \sigma_{1}$ & 2 & $5.1 \times 10^{-3}$ & $3.1 \times 10^{-4}$ \\
\hline r.m.s. bunch radius $(\mu m), \sigma_{t}$ & .15 & $4.0 \times 10^{-3}$ & $1.1 \times 10^{-3}$ \\
\hline final focus, $\beta^{*}(\mathrm{~mm})$ & 7.0 & .1 & .1 \\
\hline emittance $\left(m\right.$ rad), $\varepsilon_{n}$ & $6.1 \times 10^{-6}$ & $3.1 \times 10^{-7}$ & $1.2 \times 10^{-7}$ \\
\hline beamstrahlung parameter, $\delta$ & .09 & 1.3 & $5 \times 10^{3}$ \\
\hline quantum parameter, 9 & .09 & 1.75 & 15.8 \\
\hline$\Upsilon=2 E_{c} / 3 E$ & .087 & 6.4 & $3.6 \times 10^{3}$ \\
\hline$F(\Upsilon)$ & .89 & .062 & $1.6 \times 10^{-5}$ \\
\hline$N_{Y}$ & 2.8 & .35 & .32 \\
\hline rms energy spread, $\sigma_{E}$ & .11 & .21 & .22 \\
\hline$r$ & $3.7 \times 10^{-4}$ & .15 & 12.2 \\
\hline electron density $\left(\mathrm{cm}^{-3}\right)$ & $1.4 \times 10^{19}$ & $8.6 \times 10^{22}$ & $3.4 \times 10^{25}$ \\
\hline
\end{tabular}

tem is obtained from $P$ by dividing by the efficiency of energy transfer 
from the energy stored in the accelerator to the beam, $\eta_{3}$ and by the efficiency of energy transfer from the main energy source to the accelerator, $\eta_{2}$. The product $\eta_{2} \eta_{3}$ can easily be small, in the range $10^{-1}-10^{-3}$, and this can bring the power needed to operate the collider in the GW range. This points to the importance of reducing not only the beam power but also to develop acceleration methods and accelerator power sources with high efficiency. In other words, the efficiency is as important as the maximum accelerating field in comparing different accelerator schemes.

The normalized emittance values appearing in the table must be compared with the present state of the art, based on the use of electron storage ring optimized for producing a small emittance. These rings have been developed in connection with $\operatorname{SLC}^{(13,14,15)}$ and also as synchrotron radiation sources ${ }^{(16)}$, and Free Electron Laser sources (17). The emittance obtained in the SLC rings is on the order of $10^{-5} \mathrm{~m}$, much larger than the value needed in the low power "quantum" collider of Table 1 . In one synchrotron radiation source an emittance of about $10^{-7} \mathrm{~m}$ has been obtained in the vertical plane (16). Preliminary designs for $10^{-7}$ in both planes are being considered.

It is also interesting to notice the very high values of the electron density during the crossing. Again one must compare with values presently obtained in storage rings, of the order of $10^{13} \mathrm{~cm}^{-3}$, and in SLC ${ }^{(14)}$, of the order of $10^{18} \mathrm{~cm}^{-3}$. The "classical" collider of Table 1 is close to SLC also in this respect. 
The bunch frequency is also much higher than that of existing linacs; SLAC's frequency is a few hundred Hertz. One can also notice that the accelerating field used in $\mathrm{SLC}^{(14)}$ would lead to a length of $60+60 \mathrm{~km}$ for a $1 \mathrm{TeV}$ collider.

The final focus designed for SLC ${ }^{(14)}$ provides a $\beta^{*}$ of about 5 millimeters. Again the $\beta^{*}$ value of the second and third collider of Table 1 is much smaller. All this shows that the construction of high energy, high luminosity colliders, requires improvements over the present state of the art in many areas, like the final focus, the emittance, the accelerating field and frequency of the accelerator, understanding of very high density electron bunches. All this is particularly true for parameters which lead to the quantum regime of beamstrahlung.

Figs. 3 and 4 show, on the other hand, that the beam parameters and collision frequency needed for a $(5+5) \mathrm{TeV}, \mathscr{L}=10^{34} \mathrm{~cm}-2 \mathrm{~s}-1$ or larger, are less challenging in the quantum regime than in the classical regime. Thus the very short and small bunches considered in the $(1+1) \mathrm{TeV}$ "quantum case" of Table 1, have to be studied if one wants to move in the direction leading to colliders with even larger energy and luminosity. 


\section{Acceleration Techniques}

A number of new acceleration techniques have been studied recently and a description of the most recent work can be found, for instance, in the proceedings of the Los Angeles Workshop on New Acceleration Techniques (18). One of these techniques is the Two Beam Accelerator (TBA), proposed by A.M. Sessler (11). In the proposed TBA a low energy electron beam produces radiation, at around $1 \mathrm{~cm}$ wavelength, in a Free Electron Laser (FEL). The energy lost by the low energy beam is periodically restored by an induction linac. The one centimeter radiation is fed to a linac structure where accelerating fields of a few hundred $\mathrm{MeV} / \mathrm{m}$ can be obtained.

Recently an experiment by a a Berkeley-Livermore group, has demonstrated the possibility of using Free Electron Lasers, (FEL), as power sources at a wavelength around $1 \mathrm{~cm}$, obtaining accelerating fields up to $250 \mathrm{MeV} / \mathrm{m}$ in a linac structure ${ }^{(19)}$.

The possibility of going to still shorter wavelengths, in the $\mathrm{mm}$ or $\mu \mathrm{m}$ range, using structures like gratings, droplet arrays or an inside-out linac, has also been actively studied $(20,21)$. In these cases one could work at around $1 \mathrm{~mm}$, using again an FEL as a power source or at optical wavelengths, where lasers like an FEL or a $\mathrm{CO}_{2}$ laser could be used. In these last cases, and using very short (picosecond) pulses, one can reach accelerating fields of the order of $1 \mathrm{GeV} / \mathrm{m}$, before the accelerator walls break down or meit ${ }^{(22)}$. 
The recent developments in design and construction of superconducting cavities (23), which have established the possibility of reaching fields on the order of 5 to $10 \mathrm{MeV} / \mathrm{m}$, with quality factors of about $5 \times 10^{9}$, at temperature of about $4 \mathrm{~K}$, has also renewed the interest in the use of superconducting linacs $(2,24)$, including the energy recovery option $(25,26)$, for high energy colliders. Although the maximum accelerating field for these systems is not high, less than $60 \mathrm{MeV} / \mathrm{m}$ for $\mathrm{Nb}$ cavities (23), they might offer the possibility of high efficiency and reduced power consumption, for not too large final energies.

In this paper we want to study the possibility of substituting in a TBA the induction linac with a superconducting linac. The possible advantages are: 1) large efficiency of energy transfer to the low energy beam; 2) possibility of obtaining easily the high $e^{+}-e^{-}$collision rate needed for high luminosity.

We believe that these possible advantages, and the established availability of superconducting cavities, make this scheme worth studying. Since the superconducting cavities for increasing the LEP beam energy from 50 to about $100 \mathrm{GeV}$ work at $350 \mathrm{MHz}$, we choose this frequency for our driving linac. Low frequencies, as this, have the advantage of larger stored energy per unit length. 


\section{Energy Balance and Collider Parameters}

The basic module of our system is given in fig. 5 , and consists of an undulator of length $L_{u}$, and a length $L_{c}$ of superconducting cavities. The radiation produced in the undulator is fed to a high frequency linac (HFL) by a number of waveguides. The total module length is $L_{m^{\prime}}$ and we make the simplifying assumption that $L_{m}=L_{u}+L_{c}$.

We now want to establish the energy balance between the superconducting cavities and the high frequency structure. We will use a subscript 1 to describe the superconducting cavity characteristics, like frequency $\omega_{1}$, field $E_{1}$ etc., and subscript 2 for the HFL. The average energy stored in the superconducting cavities per unit length is $(22,27)$

$$
U_{1}=E_{1}^{2} /\left(R_{1} / Q_{1}\right) \omega_{1}
$$

where $R_{1}$ is the shunt impedance per unit length and $Q_{1}$ the quality factor. Of this energy we can transfer a fraction $\eta_{1} \eta_{2}$ to the HFL, where $\eta_{1}$ is the fraction of the energy stored in the SCCs that is given to the low energy electron beam powering the FEL, and $\eta_{2}$ is the fraction of energy produced by the FEL that is transferred to the HFL.

If $P_{1}$ is the power per unit length in the HFL, and $T_{f}$ its filling time, we obtain, by applying energy conservation to one module, the relation

$$
\eta_{1} \eta_{2} U_{1} L_{c}=P_{1} T_{f} L_{m}
$$


The HFL filling time and power per unit length are related to the structure attenuation constant, $\alpha$, quality factor, $Q_{2}$, frequency, $\omega_{2}$, shunt impedance per unit length, $R_{2}$, and the electric field, $E_{2}$, by $(22,27)$

$$
\begin{aligned}
& T_{f}=\alpha\left(Q_{2} / \omega_{2}\right) \\
& P_{1}=E_{2} 2 /\left(g^{2} \alpha R_{2}\right)
\end{aligned}
$$

where $g=2(1-\exp (-\alpha / 2)) / \alpha$. By combining eqs. (26) to (29) one can introduce $^{(28)}$ an electric field amplification factor, given by

$$
\frac{E_{2}^{2}}{E_{1}^{2}}=\eta_{1} \eta_{2} g^{2} m \frac{\left(\omega_{2} R_{2} / Q_{2}\right)}{\left(\omega_{1} R_{1} / Q_{1}\right)}
$$

where $m$ is the ratio of SCC to HFL length, $m=L_{c} / L_{m}$. Since $R / Q$ scales like $\omega$, eq. (29) shows that the ratio of the fields increases with the ratio $\omega_{2} / \omega_{1}$, hence the convenience of going to high frequency in the HFL.

We now make the further step of relating the HFL characteristics to the high energy bunch parameters determined in section 3 . For a given power flux, $P_{1}$, in the HFL, and for a given energy transfer to the beam, defined by the efficiency factor $\eta_{3}$ (=energy given to the beam per unit length/ energy stored in the HFL per unit length), we can accelerate $N_{z}$ particles in a bunch, with

$$
e N_{2} E_{2}=\eta_{3} P_{1}{ }^{\top} f
$$


and using eqs. (27) and (28)

$$
e N_{2} \omega_{2}\left(R_{2} / Q_{2}\right) g^{2}=\eta_{3} E_{2}
$$

From the field $E_{2}$ we can calculate $E_{1}$ using (30). We can also obtain $\mathrm{N}_{2}$ from (18) and write directly $E_{2}$ and $E_{1}$ as function of $E, \mathscr{L}, P, D(H)$, $\langle\varepsilon\rangle$, and the frequencies $\omega_{1}, \omega_{2}$

$$
\begin{aligned}
& \eta_{3} E_{2}=e C_{1} g^{2}\left(R_{2} \omega_{2} / Q_{2}\right)(P / E \mathscr{L})^{2}(\langle\varepsilon\rangle D H / F(\Upsilon)) \\
& \eta_{3} E_{1}=e C_{1} g\left(\frac{\omega_{1} R_{1} \omega_{2} R_{2}}{\eta_{1} \eta_{2} m Q_{1} Q_{2}}\right)^{1 / 2}(P / E \mathscr{L})^{2}(\langle\varepsilon\rangle D H / F(\Upsilon))
\end{aligned}
$$

where $C_{1}$ is given by $(20)$. Since $R / Q \approx \omega$ we see that $\eta_{3} E_{2} \approx \omega_{2}{ }^{2} / F$ and $\eta_{3} E_{1} \approx \omega_{1} \omega_{2} /(F \vee m)$. Eq. (34) shows that if we try to decrease $P$ and $D$, thus passing from the classical to the quantum regime, and we want to keep $\langle\varepsilon\rangle, \omega_{1}, \eta_{1}, \eta_{2}$ and $\eta_{3} E_{1}$ constant we have to increase $\omega_{2} /(F \vee m)$. Since $F^{-1}$ increases by a factor 15 in the example of Table 1 , and $m=L_{c} / L_{m}$ should not be reduced, by necessity the HFL frequency, $\omega_{2}$, has to increase. This entails, through (33), a welcome increase of $E_{2} \approx \omega_{2} \vee \mathrm{m}$.

In summary, eqs. (33) and (34) put retrictions on the choice of the accelerator parameters for given beam and collision conditions. Viceversa for a given accelerator system we are limited, by the same equations, in the collider performance. For a fixed, low energy SC structure, and fixed ef- 
ficiencies, the frequency and the accelerating field in the HFL come out to be much larger in the quantum regime than classical one. This puts into light one of the advantages of using a FEL to produce high frequency fields: one can reduce the wavelength by changing the undulator system and one can explore the quantum regime.

In the quantum limit, $\Upsilon>>1$, the number of particles per bunch is given by the simple expression (24), and one can use this in (33), (34). In this case there is no dependence of the products $\eta E$ on the collider energy or luminosity, which makes possible to use the same accelerator system to cover a wide range of beam energy and luminosity. This property makes the quantum regime very attractive and worth exploring in greater depth.

The length of SCC cavities is determined by the electron energy change in the undulator $\mathrm{mc}^{2}\left(\gamma_{0}-\gamma_{f}\right)$ and the electric field $E_{1}$ in the SCC

$$
m c^{2}\left(Y_{0}-\ddot{Y_{f}}\right)=e E_{1} L_{c}
$$

The electron beam that radiates energy in the undulator can be made of $b_{1}$ bunches separated by the SCC wavelength, $\lambda_{1}$. The number of particles, $N_{1}$, per bunch is determined again by energy conservation

$$
e N_{1} b_{1} E_{1}=\eta_{1} U_{1}
$$

and does not depend on the number of SCC cavities.

The peak power in the low energy beam is given by 


$$
P_{1, p}=\left(e N_{1} c / L_{B}\right) m c^{2} \gamma_{0}
$$

where $L_{B}$ is the bunch length.

Two sets of accelerators parameters for the SCC and the HFL are collected in Tables 2 and 3 . Based on the previous discussion we have considered for the HFL two cases, $\lambda=10 \mathrm{~mm}$ and $\lambda=.42 \mathrm{~mm}$, with a wavelength ratio of about 25 . The second case implies the development of a new technology for the linac structure. We have simply assumed that the characteristics of this linac can be obtained by scaling from lower frequency systems. In our present situation this cannot be fully justified and we will have to wait until more work is done to make more realistic estimates. The value $\lambda \approx .5 \mathrm{~mm}$, has been chosen also because at this wavelength there exists a $10 \mathrm{KW}$ operating $F E L(29)$, and the relative diagnostics has been developed.

Table 2: Superconducting Linac Parameters

$\begin{array}{ll}\text { frequency }(\mathrm{MHz}), \mathrm{f}_{1} & 350 \\ \text { shunt impedance }(\mathrm{G} \Omega / \mathrm{m}), R_{1} & 1.3 \times 10^{3} \\ \text { quality factor, } Q_{1} & 5 \times 10^{s} \\ \text { electric field }(\mathrm{MV} / \mathrm{m}), E_{1} & 15 \\ \text { stored energy }(\mathrm{J} / \mathrm{m}), U_{1} & 385\end{array}$




\section{Table 3: High Frequency Linac Parameters}

$\begin{array}{lll}\text { frequency }(\mathrm{GHz}), \mathrm{f}_{2} & 30 & 700 \\ \text { wavelength }(\mathrm{mm}), \lambda_{2} & 10 & .42 \\ \text { attenuation parameter, } \alpha & .5 & .5 \\ \text { filling time }(\mathrm{ns}), \mathrm{T}_{\mathrm{f}} & 11.3 & .10 \\ \text { shunt impedance }(\mathrm{G} \Omega / \mathrm{m}), \mathrm{R}_{2} & .17 & .82 \\ \text { quality factor, } \mathrm{Q}_{2} & 4100 & 848\end{array}$

The attenuation parameter has been chosen equal to .5 following an argument by Schnell ${ }^{(27)}$.

Using the data of tables 2 and 3 and assuming $\eta_{1}=.1$ and $\eta_{2}=.5$, we have from (26) for $\lambda=1 \mathrm{~cm}$ and $\lambda=.42 \mathrm{~mm}$ respectively

$$
\begin{aligned}
& P_{1}=(1.7 \mathrm{~m}) \mathrm{GW} / \mathrm{m}, \text { for } \lambda_{2}=10 \mathrm{~mm} \\
& P_{1}=(193 \mathrm{~m}) \mathrm{GW} / \mathrm{m}, \text { for } \lambda_{2}=.42 \mathrm{~mm}
\end{aligned}
$$

where $m=L_{c} / L_{m}$. These are the power levels available in the two cases and from these we can calculate the accelerating field in the HFL. 


\section{The FEL}

For a complete discussion and review of FEL properties we refer to references 30,31 ; here we summarize only the main formulae. We assume that the radiation at wavelength $\lambda$ produced by the $F E L$ is propagated in a waveguide and that a tapered undulator is used to extract power from the beam. Let $A_{0}$ be the wave amplitude at the wiggler entrance, $\omega$ its frequency, $y$ the electron energy in rest mass units. The energy change of the synchronous electron is

$$
\frac{d y}{d z}=-\left(e A_{0} a_{u} \sin \left(\psi_{r}\right)\right) / m c^{2} y
$$

where the undulator parameter $a_{u}$ is given, for a planar magnet, by the undulator magnetic field, $B_{u^{\prime}}$ and period, $\lambda_{u^{\prime}}$ as $a_{u}=e B_{u^{\prime}} \lambda_{u^{\prime}} 2^{3 / 2} \pi \mathrm{mc}^{2}$. The phase, $\psi_{r}$, in (40) is a constant provided that the condition of synchronism

$$
k_{u}=\delta k+(\omega / c)\left(1+a_{u}^{2}\right) / 2 y^{2}
$$

is satisfied. In (41) $\mathrm{k}_{\mathrm{u}}=2 \pi / \lambda_{\mathrm{u}}$, and the quantity $\delta \mathrm{k}=(\omega / \mathrm{c})-\mathrm{k}$ describes the change in the radiation wavenumber, $k$, from its value in vacuum. For a rectangular guide of height $b$ and width $a$, with $a>>b$, and for the lowest order transverse electric mode, $\delta \mathrm{k} \approx \pi \lambda / 4 \mathrm{~b}^{2}$. 
To satisfy (47) all along the undulator we have to change $\lambda_{u}$ and /or $a_{u}$ as $\gamma$ changes according to (40). It is possible to solve (40) and (41) for different types of undulators, i.e. for different choices of $\lambda_{u}$ and $a_{u}$ as a function of $z$. In the following we will consider for simplicity the case in which $a_{u}$ is kept constant and $\lambda_{u}$ changes.

To integrate (41) we also assume that $A_{0}$ is constant, so that the electron energy change per undulator is simply

$$
Y_{f}^{2}=\gamma_{o}^{2}-2 e A_{a} a_{u} L_{u} \sin \left(\Psi_{r}\right) / m c^{2}
$$

The assumption $A_{0}=$ constant can be justified since we can remove the radiation generated along the undulator and feed it to the HFL. To the field $A_{0}$ is associated a laser power, $P_{L^{\prime}}$, propagating in the waveguide

$$
P_{L}=A_{0}^{2} a b / 2 Z_{0}
$$

$Z_{0}$ being the vacuum impedance $\left(Z_{0}=377 \Omega\right)$. The energy lost by the electrons is restored by the SCC according to (35).

In addition to the synchronism condition (41) we must consider the effect of the difference in velocity between the low energy electrons used in the FEL, the radiation pulse propagating in the waveguide, and the high energy electrons in the HFL. Let us consider first the radiation group velocity, $v_{\mathcal{G}}$, and the low energy electron axial velocity, $v_{1}$; we have 


$$
\begin{aligned}
& v_{g}=c /\left(1+(\pi c / \omega b)^{2}\right)^{1 / 2} \\
& v_{1}=c\left(1-\left(1+a_{u}{ }^{2}\right) / 2 \gamma^{2}\right)
\end{aligned}
$$

The group velocity (44) has been written again for the lowest order transverse electric mode, and assuming $a>>b$. The electron velocity, $v_{1}$, will change along the module as $Y$ and $a_{u}$ change. We can define an average longitudinal velocity

$$
\left\langle v_{1} / c\right\rangle=1-S_{1}-S_{2}
$$

where

$$
\begin{aligned}
& S_{1}=\left(L_{u} / L_{m}\right)\left(1+a_{u}{ }^{2}\right) \ln \left(\gamma_{0} / \gamma_{f}\right) /\left(\gamma_{0}{ }^{2}-\gamma_{f}{ }^{2}\right) \\
& S_{3}=L_{c} / 2 L_{m} Y_{0} \gamma_{f}
\end{aligned}
$$

and $\gamma_{0}, \gamma_{f}$ are the electron energies at the entrance and exit of the undulator, and we used (35), (42) to evaluate the effect of the electron energy variation.

The difference between the radiation group velocity and the low energy electron velocity is important in our case since we are dealing with very short, a few centimeter long, electron bunches and we want to keep using the same electrons over many modules, each of length $L_{m}$. To avoid a deformation of the radiation pulse and the developments of the sideband instability ${ }^{(31)}$ we assume 


$$
v_{g}=\left\langle v_{1}\right\rangle
$$

By choosing $Y_{0}, Y_{f}$, and the ratio $L_{u} / L_{m}$ we can satisfy (49), so that in one module there is no slippage between the electrons and the radiation pulse. If we satisfy the condition (49), we would still be left with a slippage over the undulator length

$$
S_{U}=L_{U}\left\{(\lambda / 2 b)^{\left.2 / 2-S_{1}\right\}}\right.
$$

In practical cases, like the ones we will consider, this turns out to be much smaller than the bunch length, and we will assume that this effect can be neglected.

We can now use the condition (49), and the synchronism condition (41), to determine $\lambda_{u}$ and $a_{u}$ for given $Y_{0}, Y_{o} / Y_{f}, m=L_{c} / L_{m}$, and $L_{u} / L_{m}$. In this way we can design a module producing an energy exchange given by (40) and also $v_{g}=\left\langle v_{1}\right\rangle$. For our estimates we have chosen the particular parameters given in Table 4, for two cases, corresponding to radiation at 10 $\mathrm{mm}$ and $.42 \mathrm{~mm}$ wavelength.

The difference between the low energy electron and the high energy electron longitudinal velocity is given to a good approximation by $c-\left\langle v_{1}\right\rangle$. The delay per module is given by

$$
S_{H F L}=L_{m}\left(1-\left\langle v_{1}\right\rangle / c\right)
$$


Table 4: Undulators Parameters

$\begin{array}{lll}\text { radiation wavelength }(\mathrm{mm}), \lambda_{2} & 10 & .42 \\ \text { electron energy/mc }{ }^{2}, Y_{0} & 180 & 360 \\ \text { energy change, } Y_{0} / \gamma_{f} & 2 & 2 \\ \text { SCC length/module }(\mathrm{m}), & 3 & 6 \\ \text { SCC length ratio, } \mathrm{L}_{\mathrm{c}} / \mathrm{L}_{\mathrm{m}} & .2 & .2 \\ \text { module length }(\mathrm{m}), \mathrm{L}_{\mathrm{m}} & 15 & 30 \\ \text { waveguide height }(\mathrm{cm}), \mathrm{b} & 5 & 1.5 \\ \text { undulator parameter, }{ }^{\mathrm{u}} \mathrm{u} & 14.8 & 8.15 \\ \text { undulator period }(\mathrm{cm}), \lambda_{\mathrm{u}} & 120 & 120 \\ \text { undulator slippage, } \mathrm{S}_{\mathrm{u}} / \mathrm{L}_{\mathrm{u}} & 6.2 \times 10^{-6} & 1.6 \times 10^{-8} \\ \mathrm{HFL} \text { slippage, } \mathrm{S}_{\mathrm{HF}} / \mathrm{L}_{\mathrm{m}} & 5.2 \times 10^{-3} & 9.8 \times 10^{-5} \\ & & \\ \text { If (49) is satisfied this equation can also be rewritten as } & & \end{array}$

This shows that this slippage is determined by the characteristics of the waveguide, and that it is less severe at shorter radiation wavelength. The high energy electron slippage can be neglected as long as it is small compared with the low energy electron train length, which will be discussed in the next section. If it becomes of the order of the train length, then one can compensate by making the length of the waveguides joining the FEL and the HFL of variable length, or by increasing the train length. Assum- 
ing a train length of about twice the filling time of the structure, given in Table 3, one obtains that the high and low energy beams slip by the train length in about 1.3 and $1.1 \mathrm{Km}$ respectively for the $1 \mathrm{~cm}$, or the $.42 \mathrm{~mm}$ cases. Tapering the waveguide length can increase this distance, hence "supermodules", using the same low energy bunches, longer than $1 \mathrm{~km}$ can be envisaged.

Up to now we have discussed the behaviour of the synchronous particle. There are other conditions to be imposed for the stability of the motion of non synchronous particles. The most important is a limitation on the beam energy spread, that must be smaller than $(30,31)$

$$
(\Delta E / E)_{M}{ }^{2}=\frac{e A_{o} a_{u} \lambda_{u}}{\pi m c^{2} \gamma^{2}}\left\{\cos \psi_{r}-\left(\pi / 2-\psi_{r}\right) \sin \psi_{r}\right\}
$$

The FEL model that we have considered, with $a_{u}$ and $A_{0}$ constant, is a simple one. Other possibilities can be considered, like for instance allowing $A_{0}$ to grow in the undulator region, which can provide more efficient energy extraction, or a reduced undulator length. However this model gives results which are a lower limit to what can be obtained, and we will use it to obtain our simple estimates of the SCC-FEL-HFL system. 
6. The HFL

We can now combine the results of sections 4 and 5 to determine the performance of the HFL. In addition we also have to consider the condition for matching the HFL filling time to the time structure of the FEL radiation, which consists of pulses with a duration nearly equal to the electron bunch duration, $L_{b} / c$, separated by the $S C C$ period, $\lambda_{1} / c$.

If the filling time is much longer than the bunch duration

$$
T_{f} \gg L_{b} / c
$$

it is convenient to divide the beam in the FEL in $b_{1}$ bunches

$$
b_{1}=T_{f} c / L_{b}
$$

When (53) is not satisfied it is convenient to have

$$
L_{b}=T_{f} c
$$

We must also remember that the bunch length determines the energy spread in the low energy beam, and that this must remain below the limit given by (52). If for simplicity we assume that the bunch is travelling at the crest of the wave in the SCC, than the energy spread is related to the bunch length by 


$$
\Delta E / E=(1 / 2)\left(2 \pi L_{b} / \lambda_{1}\right)^{2}
$$

From the equations (33) and (34) we can calculate $E_{2}$ and $E_{1}$ and the RF power, $P_{R F}$, to be fed into the SCC's

$$
P_{R F}=\eta_{2} \eta_{3} P=\eta_{1} U_{2} m L_{T}^{f}
$$

where $L_{T}$ is the total single linac length

$$
L_{T}=E / E_{2}
$$

The energy stored in the linac, $U_{1} \mathrm{~mL}_{T}$, determines also the amount of cryogenic power, $\mathrm{P}_{\text {cryo' }}$ needed

$$
P_{\text {cryo }}=U_{1} \omega_{1} m L_{T} / Q_{2} \eta_{4}
$$

where $\eta_{4}$ is the efficiency in cooling the SCC's. 


\section{The damping rings}

To complete the discussion of our colliders we must give a description of the damping rings producing the electron and positron beams with the required phase space density. We assume that the damping rings are built as a sequence of $\mathrm{N}_{\mathrm{d}} / 2$ achromatic modules, each with two bending magnets, similar to a Chasman-Green structure (33), and that its normalized emittance, $\varepsilon_{n}$, and momentum compaction, $\alpha_{c}$, are given by

$$
\begin{aligned}
& \varepsilon_{n}=1.2 \times 10^{-11}\left(\gamma / N_{d}\right)^{3} \mathrm{~m} \mathrm{rad} \\
& \alpha_{c}=(2 / 3) \pi^{2}(\rho / R)\left(1 / N_{d}{ }^{2}\right)
\end{aligned}
$$

where $\rho$ is the bending radius and $R$ the average radius of the damping rings. Both the emittance and the momentum compaction value depend on the particular magnetic structures one assumes, but the values given in (61), (62) are reasonable approximations for a rather large class of storage rings.

The choice of the beam energy in (61) has to be done to make the intrabeam scattering effect $(34,35,36)$ small or of the same order than synchrotron radiation effects, in determining the beam emittance.

Small emittance storage rings, based on a Chasman-Green structure, have been built up to now mainly as synchrotron radiation sources. Normalized emittances of the order of about $10^{-4} \mathrm{~m}$ rad, in the horizontal 
plane, and down to $3 \times 10^{-7} \mathrm{~m}$ rad, in the vertical plane, have been obtained. Similar rings with an emittance of about $10-5 \mathrm{~m}$ rad, have been studied in great details and look feasible ${ }^{(37)}$.

Damping rings based on a FODO structure have been built for SLC reaching an emittance of about $10^{-5} \mathrm{~m}$ rad, with more than $10^{10}$ particles per bunch (15). The design of rings pushing the emittance down to $10^{-7}$ or $10^{-8} \mathrm{~m}$ rad might introduce new problems, related, for instance, to the strong chromatic and non-linear aberrations characteristics of these systems. This, in turn, might lead to a strong reduction of the useful aperture and thus injection and lifetime problems. It is likely that to solve these problems it will be necessary to introduce much tighter magnetic element tolerances and beam orbit control.

The beam energy spread and bunch length have to be chosen to produce a longitudinal phase space emittance which matches that of the collider. While the bunch length is given in Table 1, the energy spread is also related to the choice of the accelerator characteristics, in particular the beam loading coefficient, $\eta_{3}$. A larger $\eta_{3}$ produces a larger energy spread and that might make more difficult to design the system to focus the beams at the collision point. To design the storage ring we assume $\eta_{3}=2 \%$; a larger value would make the storage ring design easier and increase the collider efficiency but it is likely to produce problems with the final focus.

To evaluate the beam energy spread and bunch length in the damping ring we assume that these quantities are determined by radiation effects ${ }^{(38)}$, thus neglecting the microwave instability and the coupled bunch 
longitudinal instability (39). This cannot be justified without a full analysis of a damping ring design. We notice however that for the very short bunch length we are considering, the ring impedance driving the microwave instability can be reduced only to the vacuum impedance and that this can be reduced to about. $1 \Omega$ (37) The control of the coupled bunch instability poses a greater problem, since this is mainly determined by the low frequency part of the impedance, which in the damping ring is not likely to be reduced respect to exsisting rings, and because to obtain the high collision frequency needed in the collider, we need to fill almost all available RF buckets. Thus in the damping ring we want to achieve a large average current and a small bunch separation. To control the coupled bunch longitudinal instability under these conditions, would require a large bandwidth, ( on the order of $1 \mathrm{GHz}$ ), feedback system, and we assume that this can be done.

With these assumptions we can assume that the energy spread and the bunch length are given by (38)

$$
\begin{aligned}
& \sigma_{\varepsilon}=4.4 \times 10^{-7} \gamma / V \rho(m) \\
& \sigma_{1}=\sigma_{\varepsilon} R\left\{2 \pi E \alpha_{c} / h e V o \cos \phi_{S}\right\}^{1 / 2}
\end{aligned}
$$

The number of bunches per second that can be obtained from a system of $N_{R}$ damping rings, assuming that each bunch stays in the ring for 5 damping times, is given by 


$$
f=h N_{R} F_{f} / 5 T
$$

where $h$ is the harmonic number, $F_{f}$ is a filling factor ( $F_{f}=$ ratio of number of bunches to the harmonic number), and $T$ is the ring betatron damping time, given by ${ }^{(35)}$

$$
1 / \tau=(1 / 3)\left(r_{e} c \gamma^{3} / \rho R\right)
$$

having assumed a damping partition factor of 2 .

To have a large frequency, $f$, we can increase $F_{f}$ and $N_{R}$ or decrease T. To obtain $F_{f}$ equal to one we need very fast kicker magnets, with a rise time of the order of the RF period; present technology limits this rise time to tens of nanoseconds, which would give, for $f_{R F}=500 \mathrm{MHz}$ a filling factor, $F_{f}$, smaller than .1. A larger value of $F_{f}$, can be obtained if a. train of $b_{2}$ bunches is injected, damped and extracted simultaneously from a damping ring. Such a procedure could be limited by wake field effects in the HFL and has to be investigated.

Table 5 contains possible set of parameters for the damping rings needed for the colliders we are considering. The conclusion is that, for $F_{f} \approx .1$, the total length of the positron damping ring system is about $1.5 \mathrm{~km}, 30$ $\mathrm{km}$, and $9 \mathrm{~km}$. Moreover for the last two cases it is likely that one will need predamping in an ad-hoc fast damping ring which will have to reduce the emittance from about $10^{-3} \mathrm{~m}$, to $10^{-5} \mathrm{~m}$, before injecting in the rings giving $10^{-7} \mathrm{~m}$. 
Table 5: Damping Rings

\begin{tabular}{|c|c|c|c|}
\hline $\mathrm{E}, \mathrm{TeV}$ & 1 & 1 & 5 \\
\hline $\mathscr{L}, \mathrm{cm}^{-2} \mathrm{~s}^{-1}$ & $10^{33}$ & $10^{34}$ & $10^{34}$ \\
\hline$\langle\varepsilon\rangle$ & .08 & .08 & .08 \\
\hline $\mathrm{D}$ & 1.3 & .05 & \\
\hline$P, M W$ & 10 & 3 & 6 \\
\hline Energy, GeV & 2.5 & 2.5 & 2.5 \\
\hline \# dipoles & 64 & 180 & 240 \\
\hline dipole length, $m$ & .87 & .29 & .29 \\
\hline Bending Field, $T$ & 1 & 1 & 1 \\
\hline Bending Radius, $\mathrm{m}$ & 8.3 & 8.3 & 8.3 \\
\hline Avergage Rdius, $\mathrm{m}$ & 25 & 25 & 33 \\
\hline Normalized Emittance, $m$ rad & $5.7 \times 10^{-s}$ & $2.6 \times 10^{-7}$ & $1.1 \times 10^{-7}$ \\
\hline Momentum Compaction & $5.3 \times 10^{-4}$ & $6.8 \times 10^{-5}$ & $2.9 \times 10^{-5}$ \\
\hline Energy loss/turn, $\mathrm{KeV}$ & 111 & 111 & 111 \\
\hline Damping time, ms & 6 & 6 & 8 \\
\hline r.m.s. energy spread & $7.6 \times 10^{-4}$ & $7.6 \times 10^{-4}$ & $7.6 \times 10^{-4}$ \\
\hline$f_{R F}, M H z$ & 500 & 500 & 500 \\
\hline$V_{0}, M V$ & 3.5 & 1.2 & 1.2 \\
\hline r.m.s. bunch length, mm & 2.2 & 1.1 & .96 \\
\hline $\mathrm{f} / \mathrm{F}_{f} \mathrm{~N}_{\mathrm{R}^{\prime}} \mathrm{KHz} / \mathrm{ring}$ & 8.7 & 8.7 & 8.6 \\
\hline$N_{R} F_{f}$ & 1 & 20 & 4 \\
\hline $2 \pi R N_{R} F_{f}, m$ & 157 & 3100 & 900 \\
\hline
\end{tabular}




\section{Results and conclusions.}

We now consider the three collider cases illustrated in Table 1, and calculate the accelerator characteristics, using the results of sections 4,5 and 6. In all cases we have tried both the $30 \mathrm{GHz},(10 \mathrm{~mm})$, case and the 700 $\mathrm{GHz},(.42 \mathrm{~mm})$, case.

For the classical, $1 \mathrm{TeV}$ collider (case 1 of Table 1), it is not possible to find a satisfactory solution at $700 \mathrm{GHz}$. At this frequency, the product $\eta_{3} E_{2}$ obtained from (33) is always very large, and gives unrealistic values for the efficiency and the field. The opposite is true for the two quantum colliders at 1 or $5 \mathrm{TeV}$ (cases 2 and 3 of Table 1), where, at $30 \mathrm{GHz}$, the accelerator length and the power needed to operate the system are always very large.

A set of solutions obtained at $30 \mathrm{GHz}$ for the $1 \mathrm{TeV}$ classical collider, and at $700 \mathrm{GHz}$ for the 1 and $5 \mathrm{TeV}$ quantum colliders are given in Table 6. Both structures are assumed to be fed by a superconducting linac, with cavities having the characteristics given in Table 2 . We have also used the HFL characteristics given in Table 3 for the two cases of 30 and 700 $\mathrm{GHz}$. The undulators characteristics for these two frequencies are given in Table 4. In evaluating the total length, $L_{T}$, of each linac we have assumed a $20 \%$ empty space, corresponding to the total length of the SC cavities.

Some relevant FEL parameter are given in the Table 7 . 
Table 6: HFL Parameters

$\begin{array}{llll}E, \mathrm{TeV} & 1 & 1 & 5 \\ \mathscr{L}, \mathrm{cm}^{-2} \mathrm{~s}^{-1} & 10^{33} & 10^{33} & 10^{34} \\ D & 1.3 & .05 & .015 \\ \langle\varepsilon\rangle & .08 & .08 & .08 \\ \mathrm{P}, \mathrm{MW} & 10 & 3 & 6 \\ \mathrm{f}_{2}, \mathrm{GHz} & 30 & 700 & 700 \\ \mathrm{E}_{1}, \mathrm{MV} / \mathrm{m} & 15 & 15 & 15 \\ \eta_{3}, \% & 10 & 10 & 10 \\ \mathrm{E}_{2}, \mathrm{MV} / \mathrm{m} & 96 & 574 & 1080 \\ \mathrm{~L}_{\mathrm{T}}, \mathrm{km} & 12.6 & 2.1 & 5.5 \\ \mathrm{P}_{\mathrm{RF}}, \mathrm{MW} & 200 & 60 & 120 \\ \mathrm{P}_{\mathrm{cryo}} \mathrm{MW} & 181 & 30 & 80 \\ \mathrm{~b}_{1} \mathrm{~N}_{1} / 10_{13} & .62 & .04 & .14 \\ \mathrm{~b}_{2} & 4 & 1 & 1 \\ \eta_{1}, \% & 3.8 & .25 & .9\end{array}$

As one can see from Table 6, the quantum case and a $\mathrm{HFL}$ at $700 \mathrm{GHz}$ are very attractive. The accelerating fields are large compared to SLC, the power levels are reasonable and are balanced between RF power and cryogenic power. One can change $\eta_{3}$, and so change the ratio of RF to cryogenic power; a smaller $\eta_{3}$ leads to a larger accelerating field and larger $R F$ power, and viceversa. The $30 \mathrm{GHz}$ case, used for the classical collider, is certainly a much smaller extrapolation with respect to today techniques. 
Table 7: FEL Parameters

\begin{tabular}{|c|c|c|c|}
\hline $\mathrm{E}, \mathrm{TeV}$ & 1 & 1 & 5 \\
\hline $\mathscr{L}, \mathrm{cm}^{-2} \mathrm{~s}^{-1}$ & $10^{33}$ & $10^{33}$ & $10^{34}$ \\
\hline$\langle\varepsilon\rangle$ & .08 & .08 & .08 \\
\hline$D$ & 1.3 & .05 & .015 \\
\hline$P, M W$ & 10 & 3 & 6 \\
\hline$f_{2}, G H z$ & 30 & 700 & 700 \\
\hline$E_{1}, M V / m$ & 15 & 15 & 15 \\
\hline$\eta_{1}, \%$ & 3.8 & .25 & .9 \\
\hline$b_{1} N_{1} / 10^{13}$ & .62 & .041 & .14 \\
\hline$b_{1}$ & 4 & 1 & 1 \\
\hline$\lambda_{2}, \mathrm{~mm}$ & 10 & .42 & .42 \\
\hline$Y_{0}$ & 180 & 360 & 360 \\
\hline$\gamma_{f}$ & 90 & 180 & 180 \\
\hline$L_{c^{\prime}} m$ & 3 & 6 & 6 \\
\hline$L_{u^{\prime}} m$ & 12 & 24 & 24 \\
\hline $\mathrm{m}$ & .2 & .2 & .2 \\
\hline$a_{u}$ & 14.8 & 8.1 & 8.1 \\
\hline$\lambda_{\mathrm{u}}, \mathrm{cm}$ & 120 & 120 & 120 \\
\hline$\Psi_{\mathrm{R}}$ & $30^{\circ}$ & $30^{\circ}$ & $30^{\circ}$ \\
\hline $\mathrm{A}_{0}, \mathrm{MV} / \mathrm{m}$ & 70 & 250 & 250 \\
\hline$P_{L^{\prime}}, G W$ & 32.5 & 38.5 & 38.5 \\
\hline
\end{tabular}

However it gives a smailer accelerating field, and a larger power. 
Furthermore a classical $5 \mathrm{TeV}$ collider at $30 \mathrm{GHz}$ requires totally unrealistic characteristics. Based on these results, the $700 \mathrm{GHz}$, quantum case seems worth of further studies.

A number of important issues have not been addressed in this paper. The most important is certainly a detailed study of an accelerating structure operating at this frequency, and which can be built with the required tolerances, and used to accelerate a high power beam. Other important issues concern the phase stability of the accelerating field, and its sensitivity to errors in beam energy and undulator magnetic field. This problem has been studied for the TBA ${ }^{(39)}$ at $30 \mathrm{GHz}$; it needs to be studied for our configuration and also at $700 \mathrm{GHz}$. Still in connection with the HFL structure one has to study the radiation extraction from the FEL waveguide, its propagation through the SC cavities, and its transmission to the HFL. Again a study has been done for TBA ${ }^{(30)}$ but not for the $700 \mathrm{GHz}$ case. Transverse wake field effects in the HFL and the low energy electron beam dynamics in the FEL also need much work, before any definite statement on the viability of the system considered in this paper can be made.

To be able to evaluate if a system like the one discussed in this paper can be used as a competitive $\mathrm{TeV}$ collider requires that many difficult problems be studied, understood and solved; we feel however that the level of performance that the system can offer would justify this effort. 


\section{Acknowledgments}

One of the authors (C. P.) wishes to thank the LEP division at CERN, and in particular Prof. E. Picasso, and Drs. E. Keil and B. Zotter, for their very kind hospitality and many useful discussions during the time this work was done.

\section{References}

[1] B. Richter, Nucl. Instr. and Methods 136,47 (1976).

[2] E.Keil et al, $\mathrm{e}^{+}-\mathrm{e}^{-}$colliders, in Proc. of the second ICFA Workshop on Possibilities and Limitations of Accelerators and Detectors, $U$. Amaldi ed., Cern, Geneva (1980).

[3] B. Richter, in Laser Acceleration of particles, C. Joshi and T. Katsouleas eds., American Institute of Physics, Conf. Proc.,no.130, P. 8 (1985).

[4] U. Amaldi, Nucl. Instr. and Methods A243,312 (1986)

[5] J. Lawson, Linear collider constraints: some implications for future accelerators, Cern report CERN 85-15, (1985).

[6] C. Pellegrini, in Proceedings of the 1985 Intern. Symp. on Lepton and Photon Interactions at High Energies, Kyoto (1985).

[7] A. M. Sessler, in Laser Acceleration of Particles, American Institute of Physics Conf. Proc., vol.91, 154 (1982); also A.M. Sessler IEEE Trans. Nucl. Sci. NS $-30,3145$ (1983).

[8] R. Hollebeek, Nucl. Instr. and Meth., 184, 333 (1985).

[9] T. Himel and J. Siegrist, in ref.2, p. 602.

[10] K. Yokoya, KEK report 85-53 (October 1985). 
[11] R. Noble, Simulation of beamstrahlung for colliding $e^{+}-e^{-}$beams with negligible disruption, AAS-note 3, SLAC (1985).

[12] In ref. (4) the beamstrahlung parameter is defined to be $2 \sqrt{ } 2$ times smaller.

[13] W. K. H. Panofsky, Limiting Technologies for Particle Beams and High Energy Physics, Slac report, Slac-Pub-3735 (1985).

[14] SLC Design Handbook, Stanford Linear Accelerator Center, Stanford (1984) .

[15] A. M. Hutton et al., IEEE Trans. Nucl. Sci. 32, 1659, (1985).

[16] M. Barton, IEEE Trans. Nucl. Sci. 32, 3350 (1985).

[17] S. Krinsky, in Free Electron Generation of Extreme Ultraviolet Coherent Radiation, eds. J.M.J. Madey and C. Pellegrini, American Institute of Physics, Conf. Proc. vol. 118 (1984), p.44.

[18] Laser Acceleration of Particles, eds. C. Joshi and T. Katsouleas, American Institute of Physics, Conf. Proc. vol. 130 (1985).

[19] D.B. Hopkins et al., IEEE Trans. Nucl. Sci. 32, 3476 (1985).

[20] R. B. Palmer et al., ref. 13, p.234.

[21] N. M. Kroll, ref. 13, p. 253.

[22] see for instance P. B. Wilson, ref.13, P. 560.

[23] H. Piel, IEEE Trans. Nucl. Sci. 32, 3565 (1985).

[24] U. Amaldi, H. Lengeler, H. Piel, Linear Colliders with Superconducting Cavities, CLIC Note-15, CERN/EF 86-8 (1986).

[25] U. Amaldi, Phys. Lett. 61B, 313 (1976).

[26] M. Tigner, Nuovo Cimento 37, 1228 (1956).

[27] W. Schnell, Dissipation versus Peak power in a Classical Linac, Cern Report LEP-RF/WS/PS (1985). 
[28] W. Schnell, Consideration of a Two Beam Twin RF Scheme for powering an RF Linear Collider, Cern Report LEP-RF/WS/PS (1985).

[29] L.R. Elias et al., Nucl. Instr. and Meth. in Phys. Res., A237, $203(1985)$.

[30] N.M. Kroll, P.L. Morton and M.W. Rosenbluth, IEEE J. Quantum Electronics QE-17, 1436 (1981).

[31] D.B. Hopkins, A.M. Sessler, and J.S. Wurtele, Nucl. Instr. and Meth. in Phys. Res. 228, 15 (1984).

[32] E.J. Sternbach and A.M. Sessler, A steady State FEL:Particle Dynamics in the FEL Portion of a Two Beam Accelerator, Lawrence Berkeley Laboratory Report LBL-19939 (1985).

[33] R. Chasman and K. Green, Brookhaven National Laboratory Report BNL 50505 (1980).

[34] H. Bruck, Accelerateurs Circulaire de Particules, Presse Universitaire, Paris (1966).

[35] A. Piwinsky, in Proc. 9th Intern. Conf. on High Energy Accelerators, Stanford (1974).

[36] J.D. Bjorken and S.E. Mtingwa, Particle Accelerators, 13, 115 (1983).

[37] J. Bisognano et al., Feasibility Study of a Storage Ring for a High Power XUV Free Electron Laser, Lawrence Berkeley Laboratory Report LBL-19771 (1985).

[38] M. Sands, The Physics of Electron Storage Rings. An Introduction., in Physics with Intersecting Storage Rings, B. Touschek ed., Academic Press, New York (1971).

[39] C. Pellegrini, IEEE Tran. Nucl. Sci. NS-28, 2413 (1981). 
[40] R.W. Kuenning and A.M. Sessler, Nucl. Instr. and Meth. for Phys. Res., A243, 263 (1986).

\section{Figure Captions}

Fig. 1 Number of particles, $N$, and collision frequency, $f, v s$ beam power,

$P$, for two $(1+1) T e V$ colliders, with $\langle\varepsilon\rangle=.08$, for $D=1.3$ and $D=.05$.

Fig.2 R.m.s. bunch length, $\sigma_{1}$, and radius, $\sigma_{t^{\prime}}$ vs beam power, $P$, for two $(1+1) \mathrm{TeV}$ colliders, with $\left\langle\varepsilon_{9}=.08\right.$, for $D=1.3$ and $D=.05$.

Fig. 3 Number of particles, $N$, and collision frequency, $f, v s$ beam power,

$P$, for two $(5+5) \mathrm{TeV}$ colliders, with $\langle\varepsilon\rangle=.08$, for $D=1.3$ and $D=.05$.

Fig.4 R.m.s. bunch length, $\sigma_{1}$, and radius, $\sigma_{t}$, vs beam power, $P$, for two $(5+5) \mathrm{TeV}$ colliders, with $\langle\varepsilon\rangle=.08$, for $D=1.3$ and $D=.05$.

Fig. 5 Schematic layout of SCC-FEL-HFL system. 


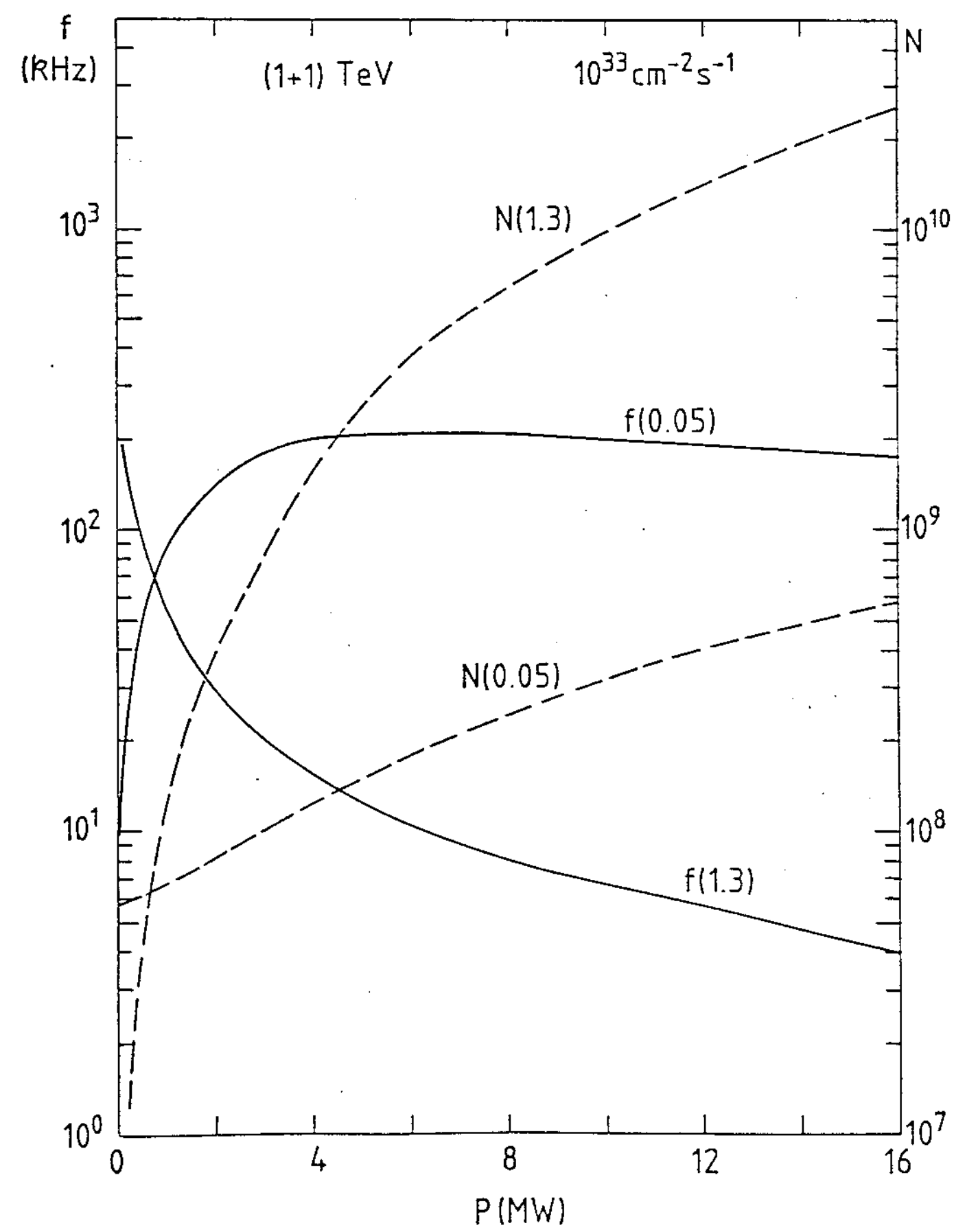

Fig. 1 Number of particles, $N$, and collision frequency, $f$, vs beam power, $P$, for two $(1+1) \mathrm{TeV}$ colliders, with $\langle\varepsilon\rangle=.08$, for $D=1.3$ and $D=.05$. 


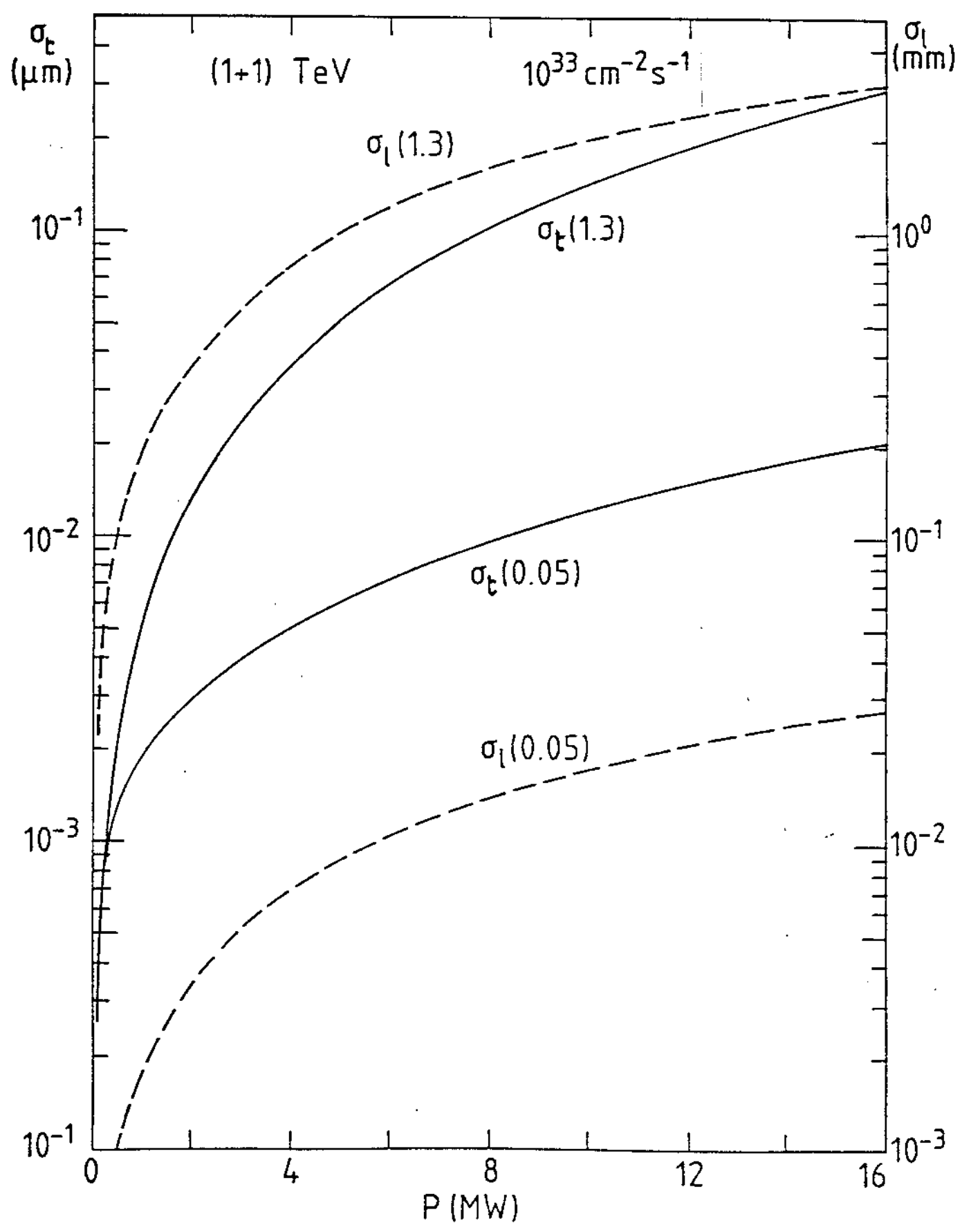

Fig. 2 R.m.s. bunch length, $\sigma_{1}$, and radius, $\sigma_{t^{\prime}}$ vs beam power, $P$, for two $(1+1) \mathrm{TeV}$ colliders, with $\langle\varepsilon\rangle=.08$, for $D=1.3$ and $D=.05$. 


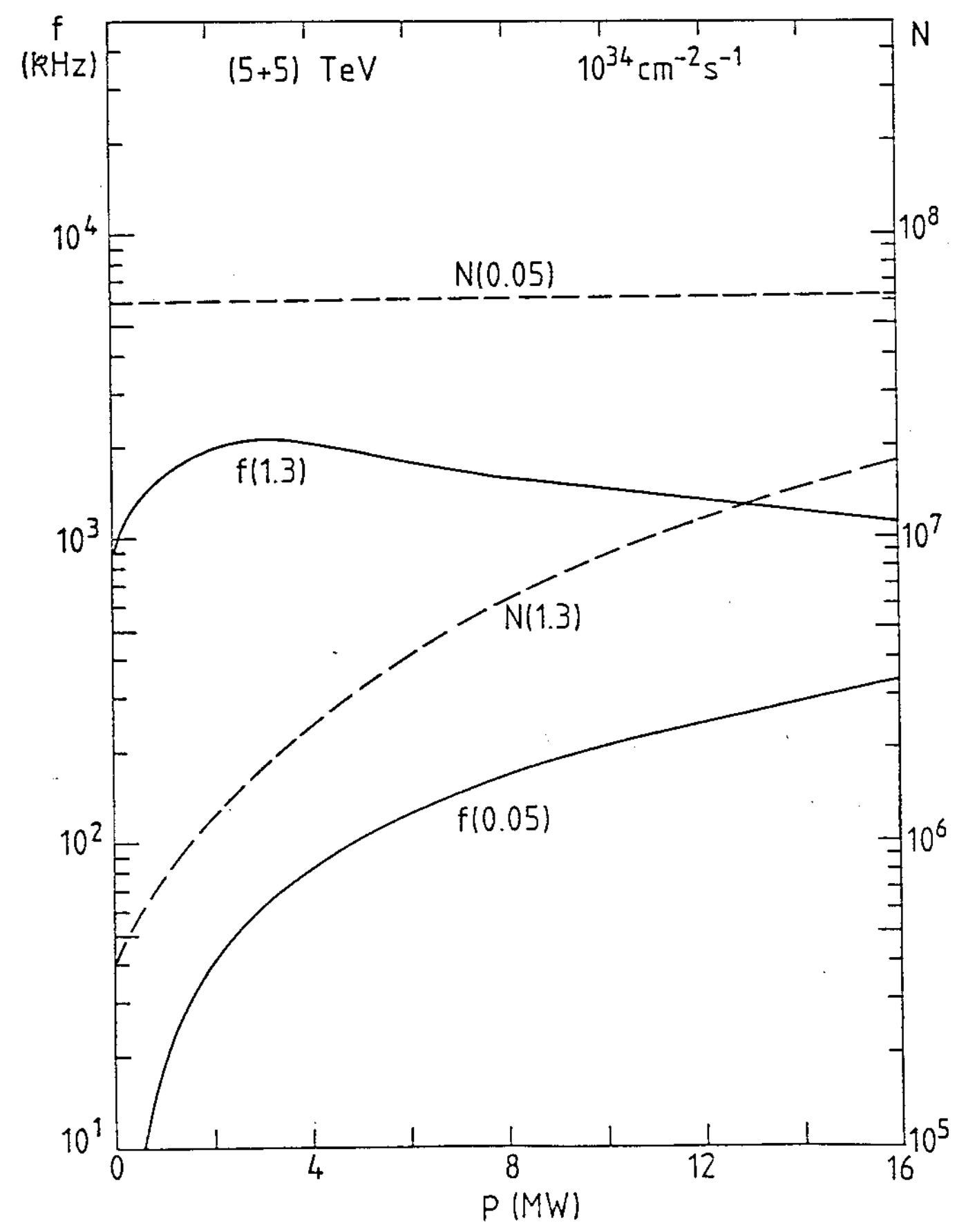

Fig. 3 Number of particles, $N$, and collision frequency, $f$, vs beam power, $P$, for two $(5+5) \mathrm{TeV}$ colliders, with $\langle\varepsilon\rangle=.08$, for $D=1.3$ and $D=.05$. 


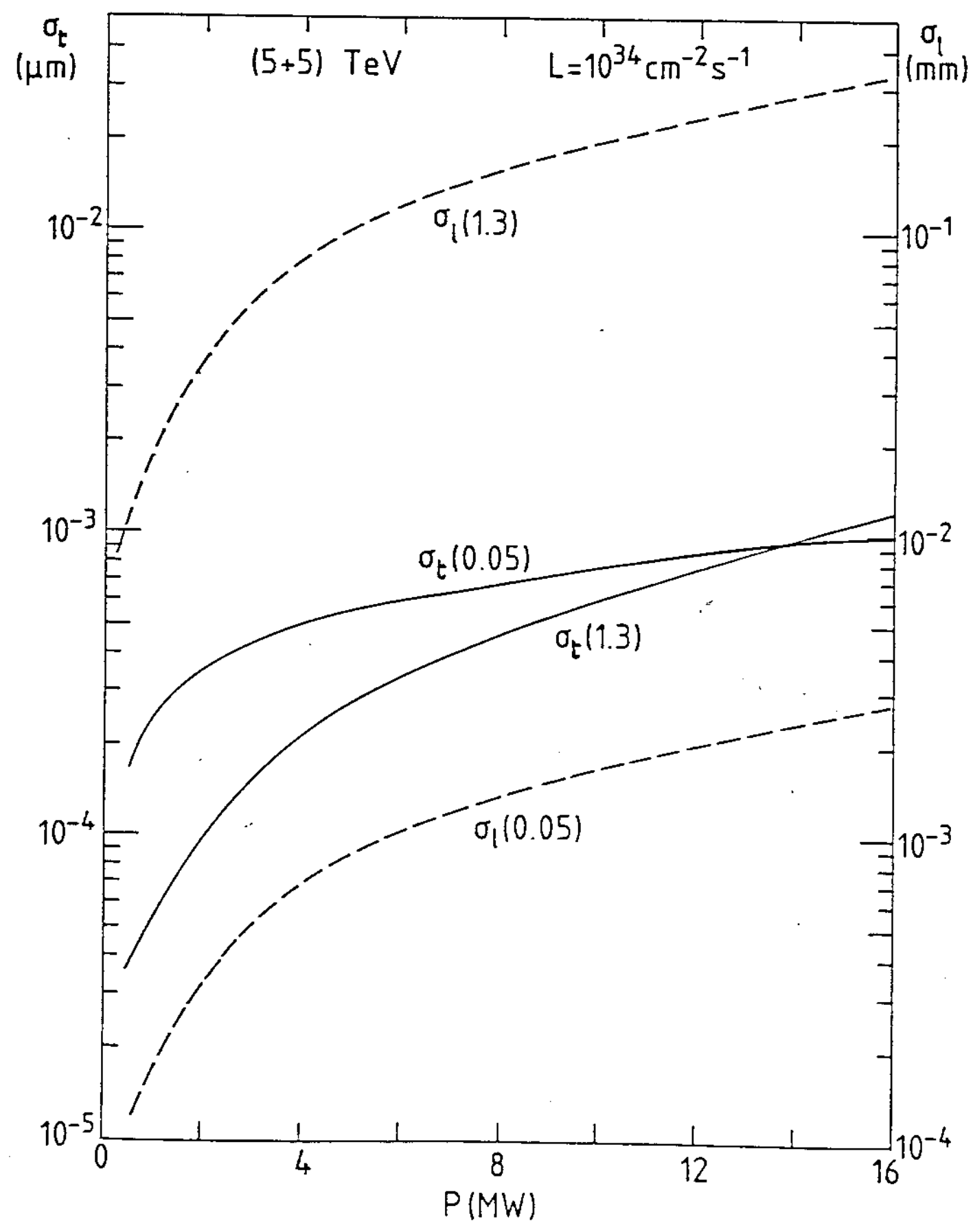

Fig. 4 R.m.s, bunch length, $\sigma_{1}$, and radius, $\sigma_{t^{\prime}} v s$ beam power, $P$, for two $(5+5) \mathrm{TeV}$ colliders, with $\langle\varepsilon\rangle=.08$, for $D=1.3$ and $D=.05$. 


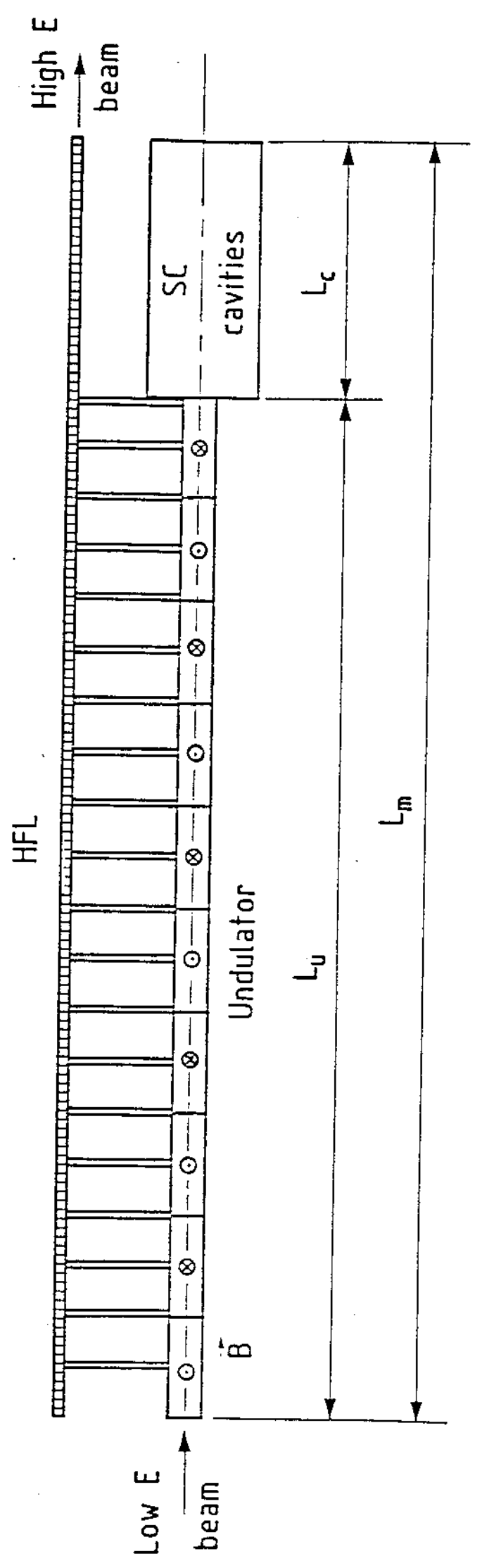

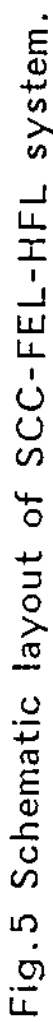

